\begin{tabular}{|c|c|}
\hline Title & Structural, magnetic, and electrical properties of Co2MnSi MgO n-GaA stunnel junctions \\
\hline Author(s) & Kawagishi, S.; Uemura, T.; Imai, Y .; Matsuda, K.-I.; Y amamoto, M. \\
\hline Citation & $\begin{array}{l}\text { Journal of A pplied Physics, 103(7), } 07 \mathrm{~A} 703 \\
\text { https://doi.org/10.1063/1.2830833 }\end{array}$ \\
\hline Issue Date & $2008-1-24$ \\
\hline Doc URL & http:/hdl.handle.net/2115/50625 \\
\hline Rights & $\begin{array}{l}\text { Copyright } 2008 \text { A merican Institute of Phy sics. This article may be downloaded for personal use only. Any other use } \\
\text { requires prior permission of the author and the A merican Institute of Physics. The following article appeared in J. A ppl. } \\
\text { Phys. } 103,07 \mathrm{~A} 703 \text { (2008) and may be found at https://dx.doi.org/10.1063/1.2830833 }\end{array}$ \\
\hline Type & article \\
\hline File Information & JAP103_07A 703.pdf \\
\hline
\end{tabular}

Instructions for use 


\title{
Structural, magnetic, and electrical properties of $\mathrm{Co}_{2} \mathrm{MnSi} / \mathrm{MgO} / \mathrm{n}$-GaAs tunnel junctions
}

\author{
S. Kawagishi, T. Uemura, ${ }^{a}$ Y. Imai, K.-I. Matsuda, and M. Yamamoto \\ Division of Electronics for Informatics, Hokkaido University Kita 14, Nishi 9, Kita-ku, \\ Sapporo 060-0814, Japan
}

(Presented on 6 November 2007; received 12 September 2007; accepted 15 October 2007; published online 24 January 2008)

\begin{abstract}
The structural, magnetic, and electrical properties of $\mathrm{Co}_{2} \mathrm{MnSi}(\mathrm{CMS}) / \mathrm{MgO}(0-3.0 \mathrm{~nm}) / n$-GaAs tunnel junctions were investigated. CMS films with $L 2_{1}$-ordered structures were grown epitaxially on GaAs. The crystallographic relations were $\mathrm{CMS}(001)[100] \| \mathrm{GaAs}(001)[110]$ when a thin $\mathrm{MgO}$ interlayer was inserted between the CMS and the GaAs, and CMS(001)[110]\|GaAs(001)[110] when the CMS film was directly grown on GaAs without a $\mathrm{MgO}$ interlayer. The CMS film without a $\mathrm{MgO}$ interlayer showed strong magnetic anisotropy consisting of uniaxial anisotropy with an easy axis of $\mathrm{CMS}[1-10](\mathrm{GaAs}[1-10])$ direction and cubic anisotropy with easy axes of CMS $\langle 110\rangle$ directions. The uniaxial anisotropy was weakened in the samples with a $\mathrm{MgO}$ interlayer. The magnetization value of the CMS film with a 3.0-nm-thick $\mathrm{MgO}$ layer was approximately $820 \mathrm{emu} / \mathrm{cm}^{3}\left(3.9 \mu_{\mathrm{B}}\right.$ / f.u. $)$ at room temperature (RT), a value slightly higher $(\sim 7 \%)$ than that of the sample without $\mathrm{MgO}$. The resistance value of the $\mathrm{CMS} / \mathrm{MgO}(3.0 \mathrm{~nm}) / n-\mathrm{GaAs}$ junction was approximately two to three orders of magnitude higher than that of the CMS $/ n$-GaAs junction at RT. The potential height and width of the tunnel barrier in the $\mathrm{CMS} / \mathrm{MgO} / n-\mathrm{GaAs}$ junction were estimated to be $0.6 \mathrm{eV}$ and $3.3 \mathrm{~nm}$, respectively. (c) 2008 American Institute of Physics. [DOI: $10.1063 / 1.2830833$ ]
\end{abstract}

\section{INTRODUCTION}

Co-based full-Heusler alloy $\left(\mathrm{Co}_{2} \mathrm{YZ}\right)$ thin films are one of the promising ferromagnetic materials for spintronic devices because of their intrinsically high spin polarization at room temperature (RT). Relatively high tunnel magnetoresistance (TMR) ratios were recently observed in magnetic tunnel junctions (MTJs) using Co-based full-Heusler alloys such as $\mathrm{Co}_{2} \mathrm{Cr}_{0.6} \mathrm{Fe}_{0.4} \mathrm{Al} \quad$ (CCFA), ${ }^{1} \mathrm{Co}_{2} \mathrm{MnSi} \quad(\mathrm{CMS}),{ }^{2,3}$ or $\mathrm{Co}_{2} \mathrm{FeAl}_{0.5} \mathrm{Si}_{0.5} .{ }^{4}$ However, in experiments on spin injection into semiconductors, relatively low injection efficiency was achieved. ${ }^{5,6}$ One possible reason is the poor interface quality between Heusler materials and semiconductors. ${ }^{7,8}$ Recently, relatively high spin injection efficiencies at RT were reported in $\mathrm{CoFe} / \mathrm{MgO} / \mathrm{GaAs}{ }^{9}$ and $\mathrm{FePt} / \mathrm{MgO} / \mathrm{GaAs} .{ }^{10}$ Insertion of a thin $\mathrm{MgO}$ layer between Heusler materials and semiconductors is therefore expected to be a useful approach for achieving high spin injection efficiency.

We recently developed fully epitaxial MTJs with a $\mathrm{Co}_{2} \mathrm{YZ}$ thin film and a $\mathrm{MgO}$ tunnel barrier, ${ }^{1,3,11}$ and demonstrated relatively high TMR ratios of $109 \%$ at RT $(317 \%$ at $4.2 \mathrm{~K}$ ) for CCFA $/ \mathrm{MgO} / \mathrm{Co}_{50} \mathrm{Fe}_{50} \mathrm{MTJs},{ }^{1}$ and $90 \%$ at $\mathrm{RT}$ $(192 \%$ at $4.2 \mathrm{~K})$ for $\mathrm{CMS} / \mathrm{MgO} / \mathrm{Co}_{50} \mathrm{Fe}_{50}$ MTJs. ${ }^{3}$ These results indicate that the spin polarization at the $\mathrm{Co}_{2} \mathrm{YZ} / \mathrm{MgO}$ interface was kept high and that the $\mathrm{Co}_{2} \mathrm{YZ} / \mathrm{MgO}$ structure is promising as an efficient spin injector into semiconductors. In previous studies, we demonstrated epitaxial growth of CCFA thin films on GaAs with a $\mathrm{MgO}$ interlayer. ${ }^{12,13}$ The structural and magnetic properties of the CCFA thin films with $\mathrm{MgO}$, however, were degraded compared with those of

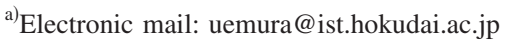

the CCFA without $\mathrm{MgO}$. In this study, we grew CMS thin films on GaAs with a $\mathrm{MgO}$ interlayer, and investigated their structural and magnetic properties. Furthermore, we characterized the electrical properties of the CMS $/ \mathrm{MgO} / n-\mathrm{GaAs}$ tunnel junctions.

\section{EXPERIMENTAL PROCEDURES}

Layer structures consisting of 400-nm-thick undoped GaAs and 100 -nm-thick $n$-GaAs $\left(\mathrm{Si}=3 \times 10^{18} \mathrm{~cm}^{-3}\right)$ were grown by molecular beam epitaxy at $580{ }^{\circ} \mathrm{C}$ on $\operatorname{GaAs}(001)$ substrates. Each sample was then capped with an arsenic protective layer and transported in air to an ultrahigh vacuum chamber equipped with magnetron sputtering cathodes and an electron beam (EB) evaporator. Prior to the growth, the arsenic cap was removed by heating the sample to $400{ }^{\circ} \mathrm{C}$. The $\mathrm{MgO}$ layer was then grown by $\mathrm{EB}$ evaporation, with the thickness $\left(t_{\mathrm{MgO}}\right)$ ranging from 0.75 to $3.0 \mathrm{~nm}$ at $200{ }^{\circ} \mathrm{C}$. Last, a 50-nm-thick CMS film was grown by rf-magnetron sputtering at $200{ }^{\circ} \mathrm{C}$. A sample without a $\mathrm{MgO}$ layer (i.e., CMS / $n$-GaAs) was also fabricated for comparison. The crystalline structures of the fabricated CMS thin films were characterized using x-ray pole figure measurements. Magnetic properties were measured using a superconducting quantum interference device magnetometer. Electrical properties of the $\mathrm{CMS} / \mathrm{MgO} / n$-GaAs tunnel junctions were measured at RT and $8 \mathrm{~K}$ using the conventional four-probe method.

\section{RESULTS AND DISCUSSION}

\section{A. Structural properties}

Figure 1 shows pole figures of 022 diffraction for the CMS thin films with $t_{\mathrm{MgO}}$ of (a) $0 \mathrm{~nm}$ and (b) $3.0 \mathrm{~nm}$. The 

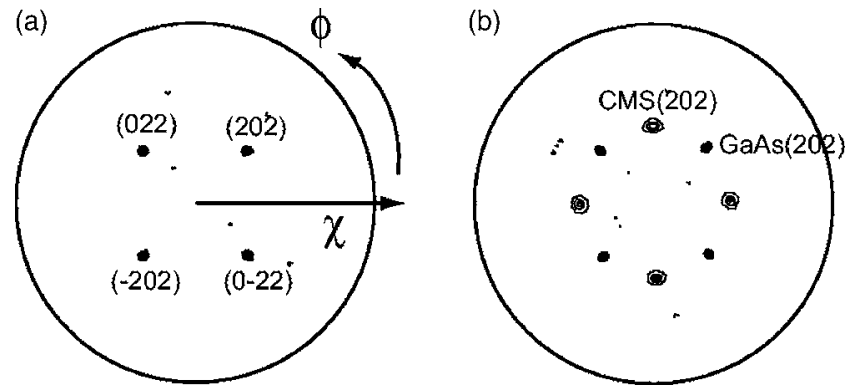

FIG. 1. Pole figure of CMS 022 diffraction in the samples with (a) $t_{\mathrm{MgO}}$ $=0 \mathrm{~nm}$ and (b) $t_{\mathrm{MgO}}=3.0 \mathrm{~nm}$. In these figures, GaAs 022 peaks are also observed due to a small lattice mismatch.

CMS 022 diffraction peaks showed fourfold symmetry with respect to the sample rotation angle $\phi$ at a tilt angle $\chi$ of $45^{\circ}$ in both samples; this was direct evidence of epitaxial growth. Here, we set the GaAs[110] direction to the origin of $\phi$. The crystallographic relations were $\mathrm{CMS}(001)[110] \| \mathrm{GaAs}(001)$ [110] (cube on cube) for the sample without $\mathrm{MgO}$, and $\operatorname{CMS}(001)[100] \| \mathrm{GaAs}(001)[110] \quad\left(45^{\circ}\right.$ rotated $)$ for the samples with a $\mathrm{MgO}$ interlayer. Furthermore, CMS 111 diffractions were observed for all the samples with a $\mathrm{MgO}$ interlayer, indicating that the CMS films had the $L 2_{1}$-ordered structure. This contrasted with previous results for the CCFA films grown on $\mathrm{GaAs}$ with a $\mathrm{MgO}$ interlayer, in which the CCFA had B2-type and/or A2-type structures, both of which are more disordered than the $L 2_{1}$ structure. $^{12,13}$

\section{B. Magnetic properties}

Figure 2 shows the magnetic hysteresis curves at RT for the samples with $t_{\mathrm{MgO}}$ of (a) $0 \mathrm{~nm}$ and (b) $3.0 \mathrm{~nm}$. The magnetic field was applied along the GaAs[110] and [1-10] directions. As shown in Fig. 2(a), the CMS film without a $\mathrm{MgO}$ interlayer showed strong magnetic anisotropy. This can be explained by a model in which (i) uniaxial anisotropy imposed by the GaAs symmetry with an easy axis of either the $\mathrm{GaAs}[110]$ or [1-10] direction and (ii) cubic anisotropy imposed by the $\mathrm{Co}_{2} \mathrm{YZ}$ with easy axes of $\mathrm{Co}_{2} \mathrm{YZ}\langle 110\rangle$ are superimposed. ${ }^{14-16}$ In our case, the easy axis direction of the uniaxial anisotropy was along the GaAs[1-10] direction. When the $\mathrm{MgO}$ interlayer was inserted, the uniaxial anisotropy was weakened [Fig. 2(b)]. For the CCFA films grown on $\mathrm{GaAs}$ with a $\mathrm{MgO}$ interlayer, however, strong uniaxial anisotropy remained even in the presence of a $\mathrm{MgO}$

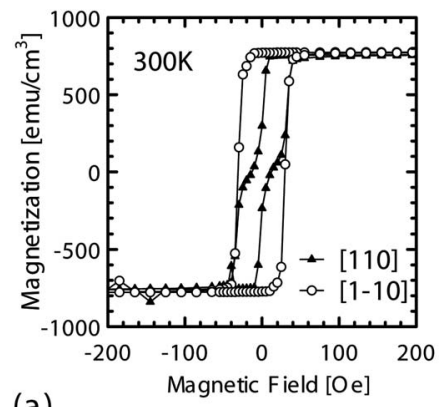

(a)

FIG. 2. Magnetic hysteresis curves at RT for the samples with (a) $t_{\mathrm{MgO}}$ $=0 \mathrm{~nm}$ and $(\mathrm{b}) t_{\mathrm{MgO}}=3.0 \mathrm{~nm}$.

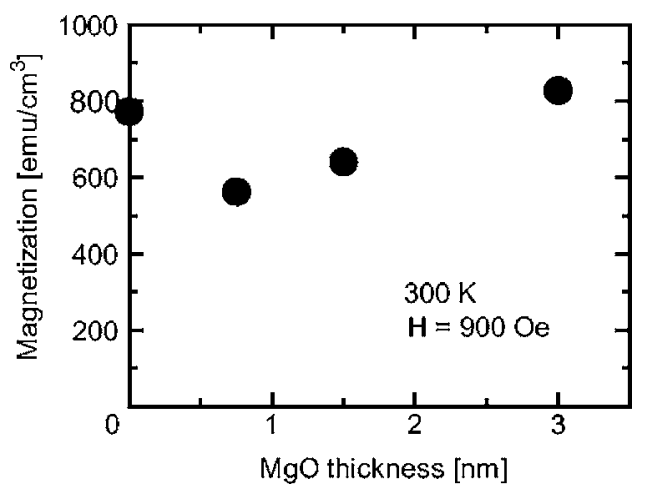

FIG. 3. Magnetization value at RT as a function of $t_{\mathrm{MgO}}$. A magnetic field of 900 Oe was applied along the GaAs[110] direction.

interlayer. $^{12,13}$ The origin of this difference between the CCFA and the CMS, though, is not yet clear.

Figure 3 shows the magnetization value $(M)$ at RT as a function of $t_{\mathrm{MgO}}$. The value of $M$ for the sample without $\mathrm{MgO}$ was approximately $770 \mathrm{emu} / \mathrm{cm}^{3}$ (equivalently $3.7 \mu_{\mathrm{B}}$ /f.u.) under a magnetic field of 900 Oe. This value corresponds to $74 \%$ of the Slater-Pauling value. When a 0.75 -nm-thick $\mathrm{MgO}$ interlayer was inserted, the $M$ decreased by approximately $29 \%$ compared with the sample without $\mathrm{MgO} . M$ increased, however, as $t_{\mathrm{MgO}}$ increased, and interestingly, $M$ for the sample with $t_{\mathrm{MgO}}=3.0 \mathrm{~nm}$ was slightly larger than that for the sample without $\mathrm{MgO}$. This result also contrasted with those obtained for the CCFA $/ \mathrm{MgO} / \mathrm{GaAs}$ structures in which the value of $M$ for the CCFA film monotonically decreased as $t_{\mathrm{MgO}}$ increased. ${ }^{12,13}$

\section{Electrical properties}

Figure 4(a) shows current $(I)$-voltage $(V)$ characteristics of the CMS/MgO $(3.0 \mathrm{~nm}) / n-\mathrm{GaAs}$ junction and $\mathrm{CMS} / n$-GaAs junction at RT. The $V$ was defined with respect to the GaAs. The junction resistance of the $\mathrm{CMS} / \mathrm{MgO} / n$-GaAs was two to three orders of magnitude higher than that of the CMS $/ n$-GaAs. This indicates that the $\mathrm{MgO}$ layer acts as a tunnel barrier. To estimate the tunnel barrier height and width in the $\mathrm{CMS} / \mathrm{MgO} / n$-GaAs, we analyzed the $I-V$ characteristics measured at $8 \mathrm{~K}$. Figure $4(\mathrm{~b})$ indicates the $I-V$ and conductance $(\mathrm{d} I / \mathrm{d} V)-V$ characteristics at $8 \mathrm{~K}$ for the $\mathrm{CMS} / \mathrm{MgO}(3.0 \mathrm{~nm}) / n$-GaAs. The conductance took the minimum value at a relatively low bias voltage of $+20 \mathrm{mV}$, indicating that the potential barrier was al-
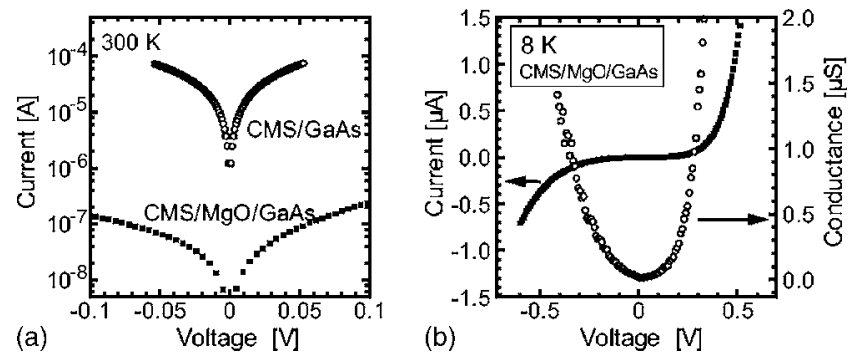

FIG. 4. (a) $I-V$ characteristics at RT for CMS/MgO $(3.0 \mathrm{~nm}) / n$-GaAs and $\mathrm{CMS} / n$-GaAs. (b) $I-V$ and $G-V$ characteristics measured at $8 \mathrm{~K}$ for CMS/ $\mathrm{MgO}(3.0 \mathrm{~nm}) / n$-GaAs. The junction size was $20 \times 100 \mu \mathrm{m}^{2}$. The bias voltage was defined with respect to $n$-GaAs. 
most symmetric; i.e., the potential barrier height at the CMS/ $\mathrm{MgO}$ interface (the energy difference between the bottom of the conduction band of the $\mathrm{MgO}$ tunnel barrier and the Fermi level of the CMS) was almost equal to that at the $n$-GaAs/MgO interface (the energy difference between the bottom of the conduction band of the $\mathrm{MgO}$ tunnel barrier and the Fermi level of the $n$-GaAs). We estimated $m^{*} \varphi$ and $d$ to be $0.6 \mathrm{eV}$ and $3.3 \mathrm{~nm}$, respectively, by fitting the $I-V$ curve based on Simmons' formula, ${ }^{17}$ where $m^{*}$ is the effective electron mass normalized by the bare electron mass, $\varphi$ is the averaged potential barrier height, and $d$ is the barrier width. The calculated value of $d$ was close to the nominal thickness of the deposited $\mathrm{MgO}$, indicating that the $\mathrm{MgO}$ layer acts effectively as a tunnel barrier.

\section{SUMMARY}

We have grown epitaxial CMS thin films with a $L 2{ }_{1}$-ordered structure on GaAs substrates with a $\mathrm{MgO}$ interlayer. The magnetization value of the CMS film with a 3.0-nm-thick $\mathrm{MgO}$ interlayer was slightly higher than that obtained in the CMS films directly grown on GaAs without a $\mathrm{MgO}$ layer. Furthermore, the resistance value of the CMS/ $\mathrm{MgO}(3.0 \mathrm{~nm}) / n$-GaAs tunnel junction was approximately two to three orders of magnitude higher than that of the $\mathrm{CMS} / n$-GaAs junction. The potential width of the $\mathrm{MgO}$ tunnel barrier calculated from Simmons' formula was close to the nominal $\mathrm{MgO}$ thickness. These results indicate that the $\mathrm{MgO}$ layer acts effectively as a tunnel barrier.

\section{ACKNOWLEDGMENTS}

We thank Professor Yasuo Takahashi for allowing us to use a cryogenic probing system for the measurement of $I$ $-V$ characteristics and for his helpful suggestions. This work was partly supported by a Grant-in-Aid for Scientific Re- search (B) (No. 18360143), a Grant-in-Aid for Scientific Research on Priority Areas (No. 19048001), a Grant-in-Aid for Scientific Research (C) (No. 19560307), and a Grant-in-Aid for Young Scientists (B) (No. 19760225) from the Ministry of Education, Culture, Sports, Science and Technology, Japan.

${ }^{1}$ T. Marukame, T. Ishikawa, S. Hakamata, K.-I. Matsuda, T. Uemura, and M. Yamamoto, Appl. Phys. Lett. 90, 012508 (2007).

${ }^{2}$ Y. Sakuraba, M. Hattori, M. Oogane, Y. Ando, H. Kato, A. Sakuma, H. Kubota, and T. Miyazaki, Appl. Phys. Lett. 88, 192508 (2006).

${ }^{3}$ T. Ishikawa, T. Marukame, H. Kijima, K.-I. Matsuda, T. Uemura, M. Arita, and M. Yamamoto, Appl. Phys. Lett. 89, 192505 (2006).

${ }^{4}$ N. Tezuka, N. Ikeda, S. Sugimoto, and K. Inomata, Jpn. J. Appl. Phys., Part 2 46, L454 (2007).

${ }^{5}$ X. Y. Dong, C. Adelmann, J. Q. Xie, C. J. Palmstrøm, X. Lou, J. Strand, P. A. Crowell, J.-P. Barnes, and A. K. Petford-Long, Appl. Phys. Lett. 86, 102107 (2005).

${ }^{6}$ M. C. Hickey, C. D. Damsgaard, I. Farrer, S. N. Holmes, A. Husmann, J. B. Hansen, C. S. Jacobsen, D. A. Ritchie, R. F. Lee, G. A. C. Jones, and M. Pepper, Appl. Phys. Lett. 86, 252106 (2005).

${ }^{7}$ S. Picozzi, A. Continenza, and A. J. Freeman, J. Appl. Phys. 94, 4723 (2003).

${ }^{8}$ K. Nagao, Y. Miura, and M. Shirai, Phys. Rev. B 73, 104447 (2006).

${ }^{9}$ X. Jiang, R. Wang, R. M. Shelby, R. M. Macfarlane, S. R. Bank, J. S. Harris, and S. S. P. Parkin, Phys. Rev. Lett. 94, 056601 (2005).

${ }^{10}$ A. Sinsarp, T. Manago, F. Takano, and H. Akinaga, Jpn. J. Appl. Phys., Part 2 46, L4 (2007).

${ }^{11}$ S. Hakamata, T. Ishikawa, T. Marukame, K.-I. Matsuda, T. Uemura, M. Arita, and M. Yamamoto, J. Appl. Phys. 101, $09 J 513$ (2007).

${ }^{12}$ T. Uemura, T. Yano, K.-I. Matsuda, and M. Yamamoto, J. Magn. Magn. Mater. 310, e696 (2007).

${ }^{13}$ T. Yano, T. Uemura, K.-I. Matsuda, and M. Yamamoto, J. Appl. Phys. 101, 063904 (2007).

${ }^{14}$ W. H. Wang, M. Przybylski, W. Kuch, L. I. Chelaru, J. Wang, Y. F. Lu, J. Barthel, H. L. Meyerheim, and J. Kirschner, Phys. Rev. B 71, 144416 (2005).

${ }^{15}$ A. Hirohata, H. Kurebayashi, S. Okamura, N. Tezuka, and K. Inomata, IEEE Trans. Magn. 41, 2802 (2005).

${ }^{16}$ T. Ambrose, J. J. Krebs, and G. A. Prinz, Appl. Phys. Lett. 76, 3280 (2000).

${ }^{17}$ J. G. Simmons, J. Appl. Phys. 34, 1793 (1963). 